\title{
An evaluation of linear instability waves as sources of sound in a supersonic turbulent jet
}

\author{
Kamran Mohseni ${ }^{\text {a) }}$ \\ Division of Engineering and Applied Science, California Institute of Technology, Pasadena, California 91125 \\ Tim Colonius \\ Division of Engineering and Applied Science, California Institute of Technology, Pasadena, California 91125 \\ Jonathan B. Freund ${ }^{\text {b) }}$ \\ Department of Mechanical and Aerospace Engineering, University of California, Los Angeles, Los Angeles, \\ California 90095
}

(Received 13 May 2002; accepted 28 June 2002; published 5 September 2002)

\begin{abstract}
Mach wave radiation from supersonic jets is revisited to better quantify the extent to which linearized equations represent the details of the actual mechanism. To this end, we solve the linearized Navier-Stokes equations (LNS) with precisely the same mean flow and inflow disturbances as a previous direct numerical simulation (DNS) of a perfectly expanded turbulent $M=1.92$ jet [Freund et al., AIAA J. 38, 2023 (2000)]. We restrict our attention to the first two azimuthal modes, $n=0$ and $n=1$, which constitute most of the acoustic field. The direction of peak radiation and the peak Strouhal number matches the DNS reasonably well, which is in accord with previous experimental justification of the linear theory. However, it is found that the sound pressure level predicted by LNS is significantly lower than that from DNS. In order to investigate the discrepancy, individual frequency components of the solution are examined. These confirm that near the peak Strouhal number, particularly for the first helical mode $n=1$, the amplification of disturbances in the LNS closely matches the DNS. However, away from the peak frequency (and generally for the azimuthal mode $n=0$ ), modes in the LNS are damped while those in the DNS grow at rates comparable to those at the peak Strouhal number. (C) 2002 American Institute of Physics. [DOI: 10.1063/1.1501545]
\end{abstract}

\section{INTRODUCTION}

There is a considerable body of evidence connecting the dynamics of large-scale structures in turbulent free shear layers to linear instability waves. ${ }^{1-3}$ Theoretical $^{4}$ and experimental ${ }^{5}$ evidence also suggests that the noise made by the large structures dominates the noise from perfectly expanded supersonic jets. The mechanism for the radiation is thought to be the generation of Mach waves due to the supersonic advection (relative to the ambient) of the structures.

Noise models based on these ideas have been developed for mixing layers ${ }^{6}$ and jets ${ }^{4}$ using matched asymptotic expansions. This approach matches near-field solutions of the linearized equations for slowly spreading flow to solutions of the wave equation in the far field. The theory correctly predicts the peak Strouhal number and noise directivity from the most amplified linear mode when compared to corresponding jet data. ${ }^{5,7}$

The noise amplitude cannot be predicted by linear theory alone, as it is directly proportional to the amplitude of the turbulent fluctuations at the nozzle lip that are presumably themselves the result of nonlinear processes. In order to scale the far field data, Tam and $\mathrm{Chen}^{8}$ consider a single instability

\footnotetext{
${ }^{a)}$ Present address: Department of Aerospace Engineering Sciences, University of Colorado, Boulder, CO 80309-0429.

${ }^{b}$ Present address: Theoretical and Applied Mechanics, University of Illinois at Urbana-Champaign, Urbana, IL 61801.
}

wave at any given frequency as representative of the energy carrying wave component of the turbulence, neglecting the continuous spectrum of convected disturbances. Then a uniformly distributed stochastic excitation seeds the instabilities at the nozzle lip region, and the amplitude of this excitation is set to match the turbulent kinetic energy of the flow at the nozzle lip.

While such calculations provide a framework for prediction, they do not provide for a quantitative evaluation of the theory. Indeed, such an evaluation has not been possible in the past, due to the daunting task of simultaneously measuring the incident turbulent fluctuations at the nozzle lip and the far-field sound. An alternative, albeit restricted to relatively low Reynolds number, is direct numerical simulation (DNS). Recently, Freund, Lele, and Moin ${ }^{9}$ computed a turbulent jet at Mach number $M=1.92$. Though the Reynolds number was low $(\operatorname{Re}=2000)$, it was shown that the directivity was similar to higher Reynolds number jets with similar convective Mach numbers. While such direct numerical simulations are themselves forced with an incident turbulent field (which is of necessity somewhat artificial, it not being possible to fully simulate the interior of the nozzle), the fluctuation fields at the inflow, as functions of space and time, are completely determined. Therefore they can be used as input to linear theory, and the predictions can be compared with the acoustic field directly determined in the DNS, 
thereby providing a detailed quantitative evaluation of the theory.

In principle, the comparison between DNS and linear theory could be obtained using adjoint-based methods to project the DNS data onto the most unstable linear mode, at each frequency, and to subsequently evolve that mode according to the matched asymptotic expansion (MAE) theory discussed above. However, construction of the adjoint eigenfunctions within the MAE framework of the models is currently an unmet challenge. We consider in the present paper the alternative of solving the full initial-boundary value problem for the linearized Navier-Stokes (LNS) equations and comparing the acoustic field so obtained to the DNS calculations discussed above. By using precisely the same inflow disturbances, we provide a direct evaluation of the linear theory of Mach wave radiation from linear instability waves for the first time.

Moreover, the comparison of LNS and DNS provides an assessment of the importance of nonlinearity in determining the amplitude of the radiation. We must note at the outset that it will not be possible to discriminate between a failure of linear theory to correctly predict the evolution of disturbances in the near field, and a failure of linear theory because of nonlinear sound generation mechanisms (such as nonlinear interactions that would comprise the "self-noise" part of Lighthill's source). In other words, the linear predictions in the present work are not necessarily the same as the linear noise source mechanism often called "shear noise" where fluctuations (potentially themselves the result of nonlinear processes) interact with the mean flow to create sound.

In the next section, the computational techniques are described. A more detailed account of the methods and issues is available elsewhere. ${ }^{10}$ Results are presented and discussed in Sec. III, and a summary of our conclusions is given in Sec. IV.

\section{COMPUTATIONAL TECHNIQUE}

The linearized Navier-Stokes equations were solved directly using the mean flow quantities and inflow boundary conditions, including an identical specification of incoming turbulent disturbances, as in the Freund et al. ${ }^{9}$ simulation. Derivatives are computed using a sixth-order compact scheme $^{11}$ in axial and radial directions and a Fourier spectral method in the azimuthal direction. A fourth-order RungeKutta algorithm advanced the solution in time. The coordinate singularity at $r=0$ is treated with the method proposed by Mohseni and Colonius. ${ }^{12}$ A fourth-order compact Padé mid-point formula was used to interpolate the flow data onto the radial grid of the LNS calculations which had the same spacing but was staggered from the DNS grid as necessitated by the centerline treatment.

In the DNS, special inflow conditions ${ }^{13}$ were used to model the behavior of the shear layer a short distance downstream of the nozzle. The inflow data came from an auxiliary DNS computation of a streamwise periodic (temporally evolving) jet, ${ }^{14}$ which had a streamwise period of $21 R$, where $R$ is the jet radius at the inlet. In order to decorrelate the turbulence of the incoming flow, the amplitude of the

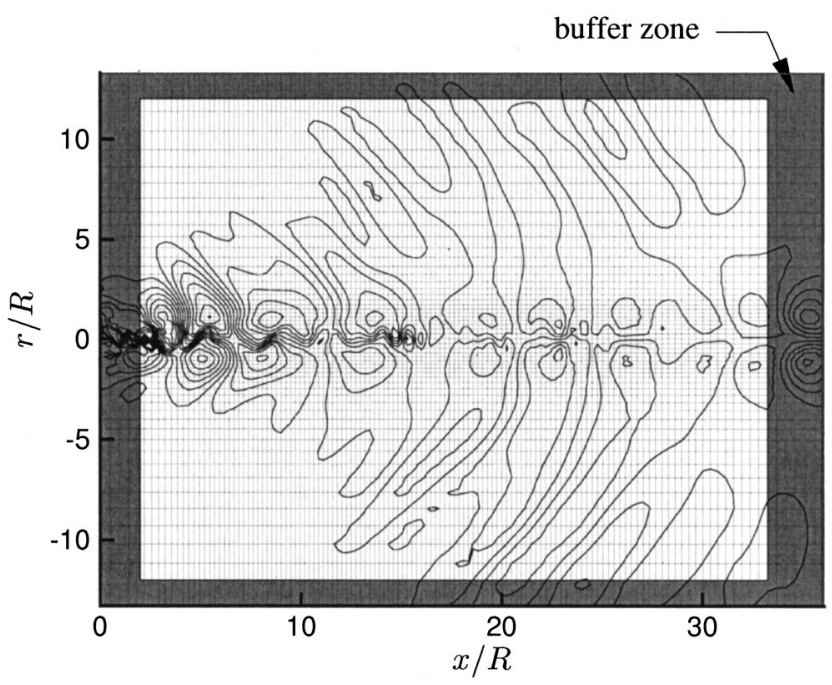

FIG. 1. Computational domain and buffer zones.

spectral components of the incoming disturbances were randomly jittered by as much as $5 \%$ of their amplitude. The decorrelation of the small scale (high frequency) turbulence statistics within the computational domain was verified $a$ posteriori. For some of the low frequency results presented in this paper, remnants of the $21 R$ periodicity remain in the DNS data. This is expected since free shear layers that are known to be sensitive to initial conditions, and a long distance is required for the large scales to be completely decorrelated. In the LNS calculations, any correlation of the incident turbulent persists indefinitely. We note that while our results are affected by this correlation, the conclusions remain valid since we are comparing the relative evolution of two flows with identical inflow disturbances.

The flow parameters in the LNS calculations were set as in the DNS calculation,

$$
\begin{aligned}
& \operatorname{Pr}=0.7, \quad \frac{T_{\infty}}{T_{j}}=0.89, \\
& \operatorname{Re}_{j}=\frac{\rho_{j} U_{j} D}{\mu_{j}}=2000, \quad M=\frac{U_{j}}{a_{j}}=1.92 .
\end{aligned}
$$

The isentropic convective Mach number ${ }^{15}$ for these conditions was $M_{c}=0.99$, the momentum thickness of the incoming shear layer was $0.1 R$, and the computational domain extended $13.3 R$ in the radial direction and $36 R$ in the axial direction; see Fig. 1. This Reynolds number is obviously lower than most laboratory jets. However, it is in this lowReynolds-number limit that linear instability waves would be expected to best represent the flow. The differences we note are only expected to be more substantial at higher Reynolds numbers. Likewise, the initial shear layer momentum thickness, $0.1 R$, is considerably larger than most laboratory jets, which will affect the stability of different frequency modes. However, our conclusions will still be general since we are comparing two jets with this same thickness. Results are expected to be insensitive to the Mach number as long as the instability waves have phase velocities that are supersonic relative to the ambient. 


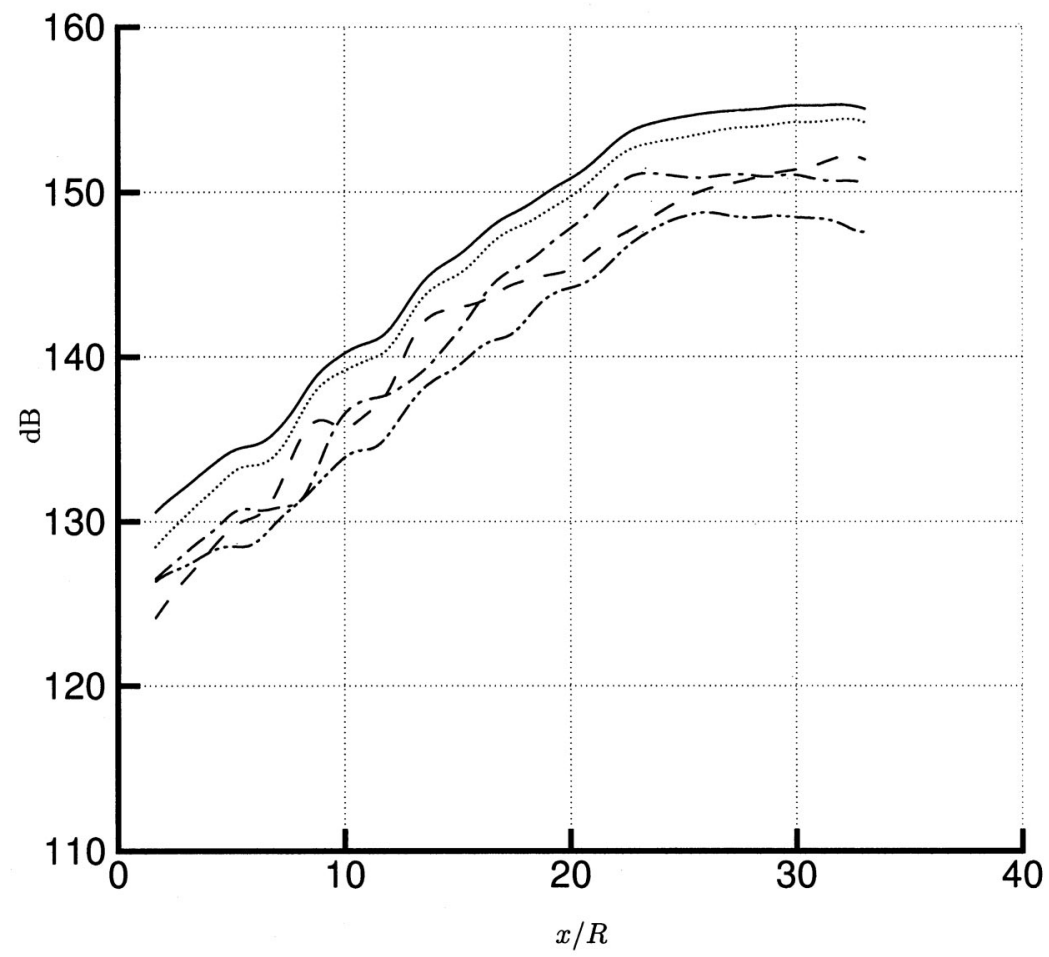

FIG. 2. SPL of the DNS at $r=12 R$ : - total; $----n$ $=0$ mode zero; $-.-n=1$ mode; $\cdots \cdots \cdots$ both $n=0$ and 1 modes; $-\cdots-$ all other modes.
The computational mesh for the DNS calculation had $640 \times 270 \times 128$ points in the axial, radial, and azimuthal directions, respectively. We found that in LNS calculations full resolution in the axial direction and half resolution in the radial direction provided results that were essentially identical to those computed with the DNS resolution. Since the acoustic field from DNS was dominated by the first two azimuthal modes (see below) we use only 4 points in the azi- muthal direction, noting that higher azimuthal modes are completely decoupled in linear computations.

\section{RESULTS}

\section{A. Sound pressure level}

We begin by comparing the sound pressure level (SPL) of the DNS and LNS data at $r=12 R$, which is the maximum

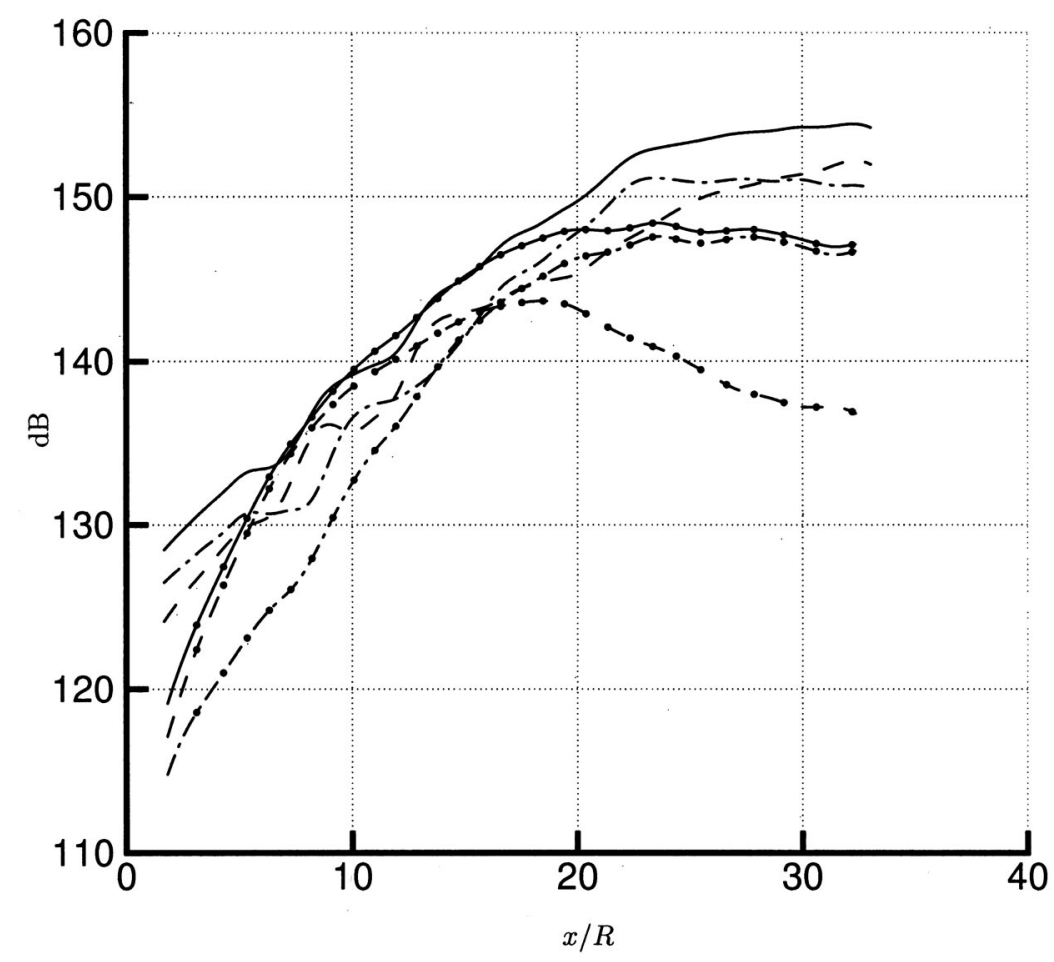

FIG. 3. SPL at $r=12 R$. For DNS: - sum of modes $n=0$ and $1 ;---n=0 ;--n=1$, and for LNS: - sum of modes $n=0$ and 1 ; 


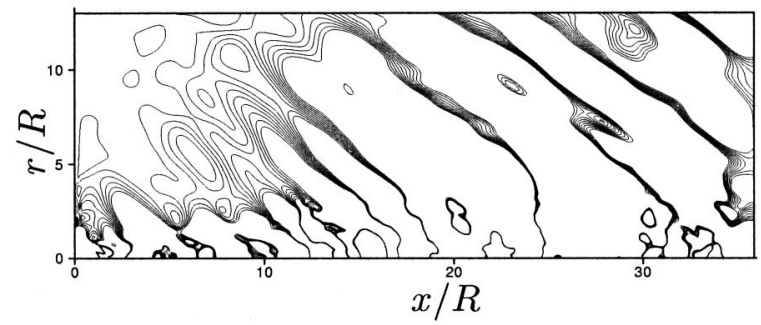

(a) DNS, $n=0$

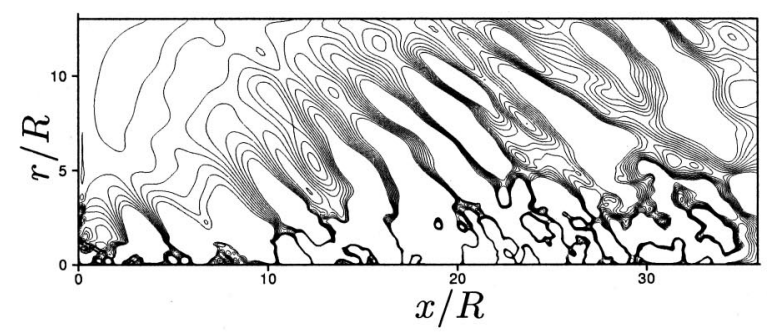

(c) DNS, real part of $n=1$

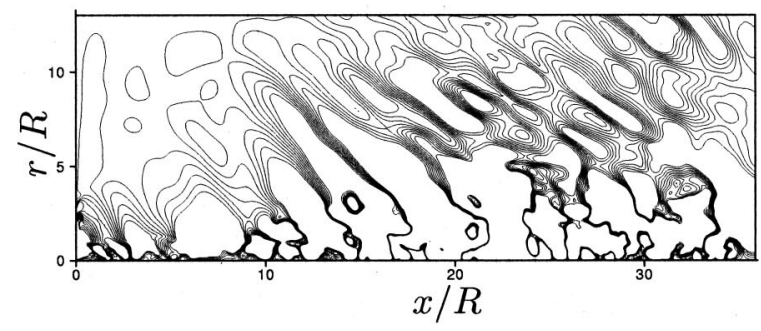

(e) DNS, imaginary part of $n=1$

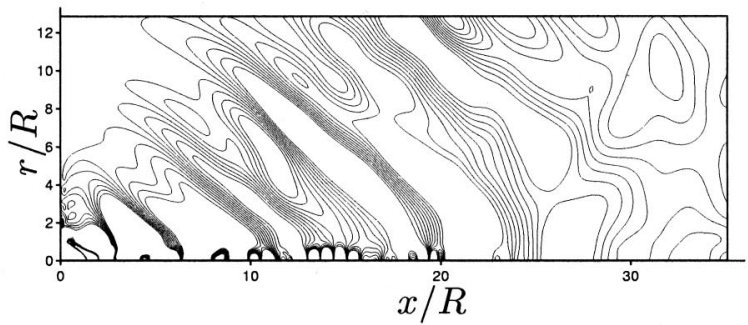

(b) LNS, $n=0$

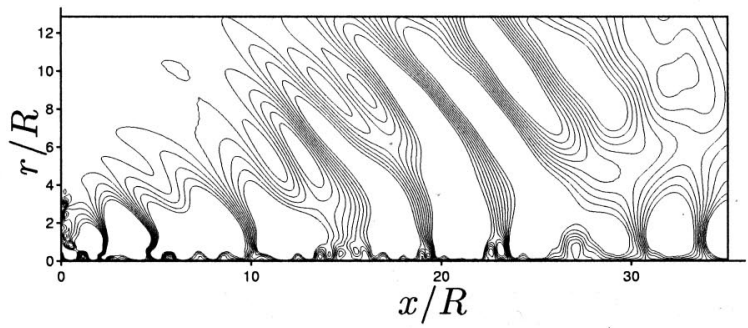

(d) LNS, real part of $n=1$

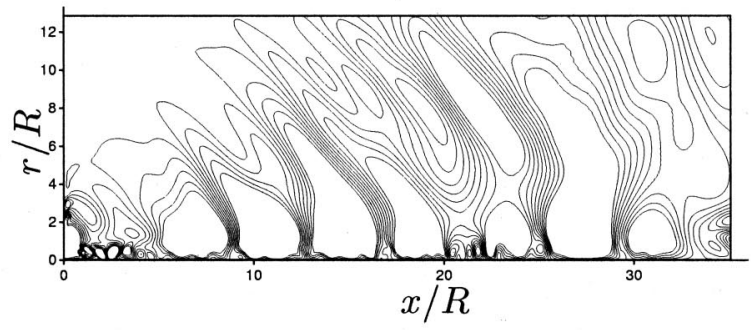

(f) LNS, imaginary part of $n=1$

FIG. 4. Instantaneous perturbation pressure field from DNS and LNS at time $222 R / a_{\infty}$, normalized with $\rho_{\infty} a_{\infty}^{2}$. Ten contour levels between -0.00225 and 0.00225 are plotted.

extent of the physically realistic (nonboundary-zone) portion of the DNS. The results of this computations are presented in Figs. 2 and 3.

There are several features to note in these figures. First, the acoustic field of the DNS data is dominated by modes zero and one. When all other modes are excluded, the total SPL is reduced by only $1.5 \mathrm{~dB}$. This confirms the predictions from linear stability theory that the acoustic field of cold jets is dominated by the first two modes. ${ }^{7}$ Because of this, we limit our attention in what follows to only the first two modes. Second, in agreement with predictions from linear stability theory, ${ }^{7}$ the azimuthal mode $n=1$ in the LNS calculation contributes the most to the total SPL and is clearly the dominant part of the generated noise. However, the same trend is not observed in the DNS data. The maximum SPL of the DNS data for $n=0$ alone is actually higher than that of mode $n=1$ and cannot be ignored, while in the LNS calculations considering only $n=1$ provides a reasonable estimate for the total SPL. Contrary to the LNS calculations, the radiation from mode $n=0$ in the DNS peaks further downstream than it does for mode $n=1$. This can be interpreted to mean that the apparent location of the sound sources of mode $n=0$ of the DNS calculation is concentrated further away from the nozzle.

In both LNS and DNS, the acoustic field is highly directional. For $n=1$ the general directivity profile is reasonably well captured. Using this mode alone in a strictly linear model would provide a reasonable prediction of the relative directivity. However, its amplitude is underestimated by $4 \mathrm{~dB}$ at $r=12 R$. The agreement is poor for $n=0$, where LNS data are less intense by as much as $15 \mathrm{~dB}$. Because azimuthal mode $n=1$ contributes the most to the total SPL of the LNS calculations, the shape of the total directivity of the LNS data is qualitatively similar to the DNS though its level is underestimated by as much as $8 \mathrm{~dB}$ at $r=12 R$. There is clearly something missing from the linear solution.

Note that these amplitude comparisons are performed outside the jet, but only at $r=12 R$. Shocks in the sound field $^{9}$ will increase dissipation of the noise so one might expect a somewhat better agreement at a larger distance from the jet, but the $8 \mathrm{~dB}$ difference at $r=12$ points to a clear problem with the source model. 


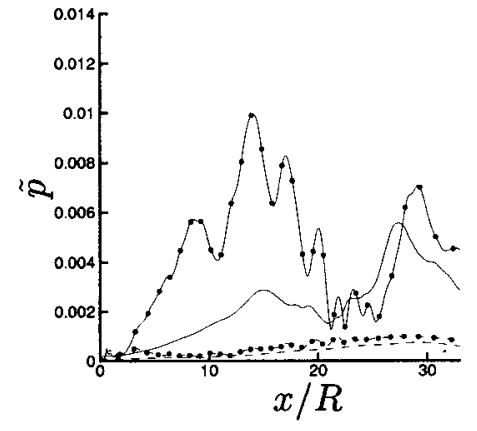

(a)

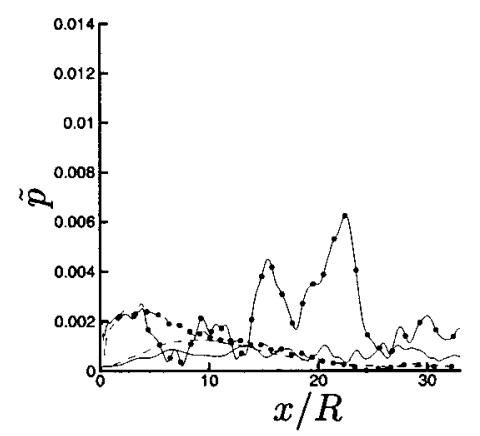

(d)

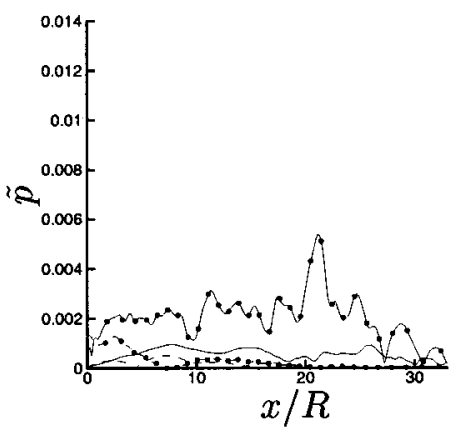

(g)

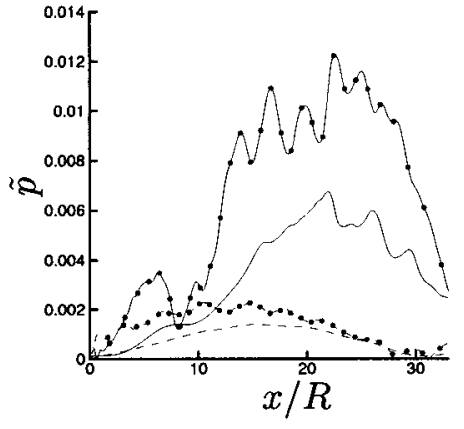

(b)

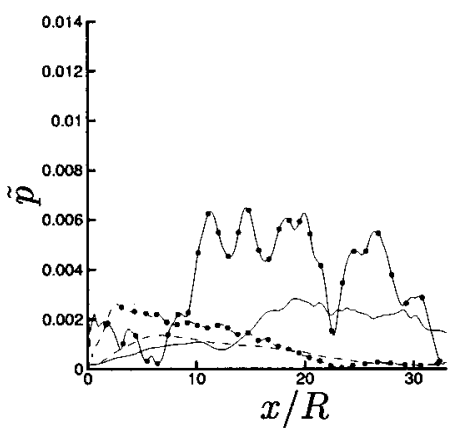

(e)

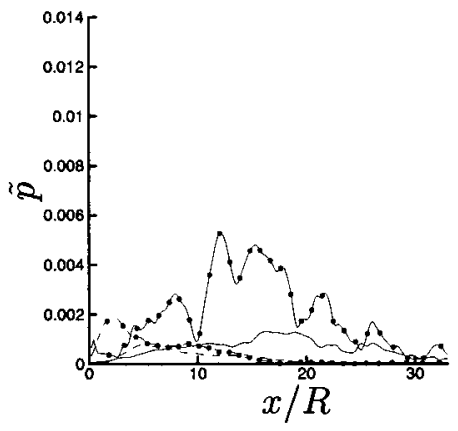

(h)

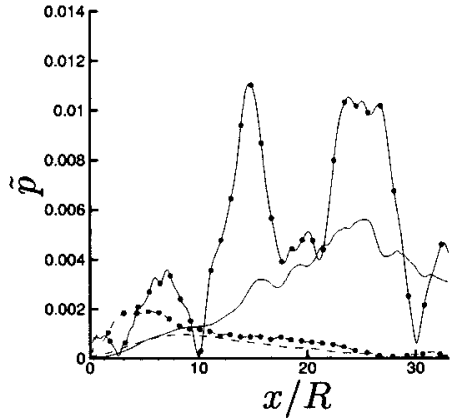

(c)

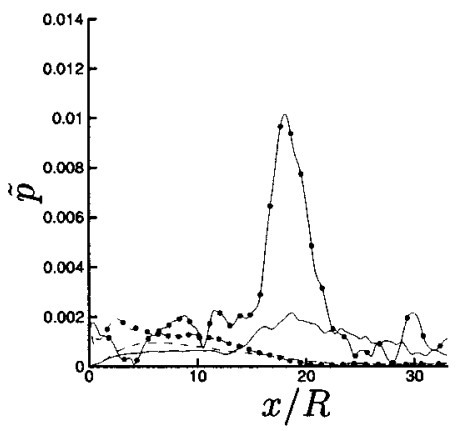

(f)

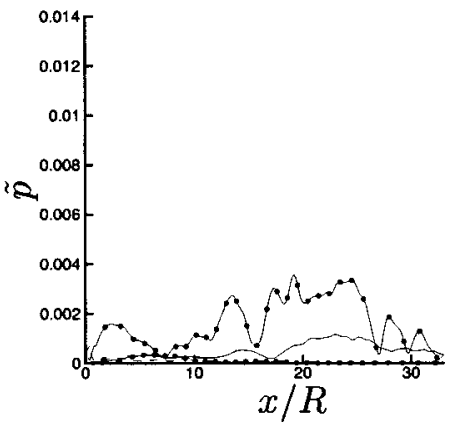

(i)

FIG. 5. Amplitude of the pressure Fourier coefficient for $n=0$ : (a) $\mathrm{St}=0.048$; (b) $\mathrm{St}=0.095$; (c) $\mathrm{St}=0.143$; (d) $\mathrm{St}=0.191$; (e) $\mathrm{St}=0.238$; (f) $\mathrm{St}=0.286$; (g) $\mathrm{St}=0.333$; (h) $\mathrm{St}=0.381$; (i) $\mathrm{St}=0.429$. The lines indicate: - $-\mathrm{DNS}$ at $r=1 R$; --@- LNS at $r=1 R$; $\longrightarrow$ DNS at $r=4 R$; ---- LNS at $r=4 R$.

\section{B. Instantaneous fields}

Instantaneous pressure fields of the DNS and LNS data for $n=0$ and 1 are shown in Fig. 4. For both $n=0$ and $n$ $=1$ the two solutions agree close to the inflow boundary. This region extends further downstream for $n=1$ than it does for $n=0$ (as described in the next section), which is consistent with the better match in amplitude discussed in the previous section. As expected, further downstream we see small scales in the DNS data that are absent in the LNS data.

It seems that the highest amplitude Mach wave radiation for $n=0$ of the LNS data originates from an area close to the inflow boundary (close to the nozzle exit) while for $n=1$ it radiates primarily from a region around $x \approx 7 R$ and extends beyond the end of the potential core. While this difference in source position is not so significant for far-field directivity, it has a substantial impact on directivity at $r=12 R$, as seen in
Fig. 3, where the SPL of the zero azimuthal mode of the LNS data at $r=12 R$ peaks earlier than that of mode one.

Thus LNS computations provide a picture consistent with linear stability theory. On the other hand, the DNS appears to have significant contributions from both the shear layer region, and from a region near the end of the potential core.

\section{Amplification of individual frequency components}

The last two sections illustrated clear differences between the LNS and DNS. What remains unclear is whether the discrepancies are due to the generation of higher frequencies in the DNS (which despite its low Reynolds number does have broadband turbulence spectra) or whether there is error even at low frequencies. To examine this, the DNS and 


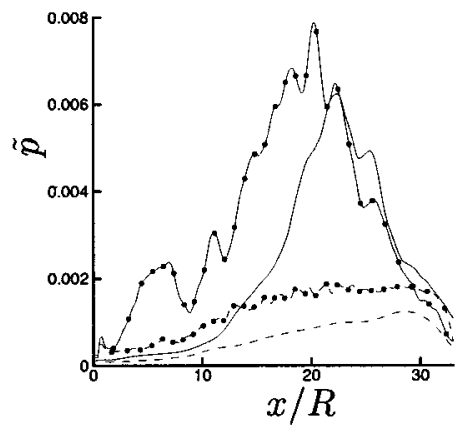

(a)

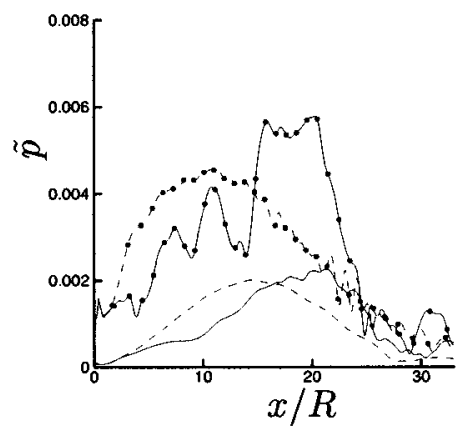

(d)

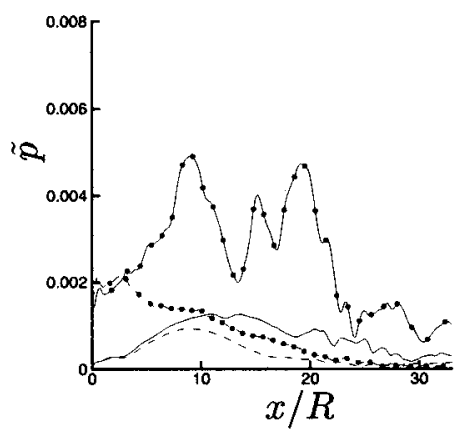

(g)

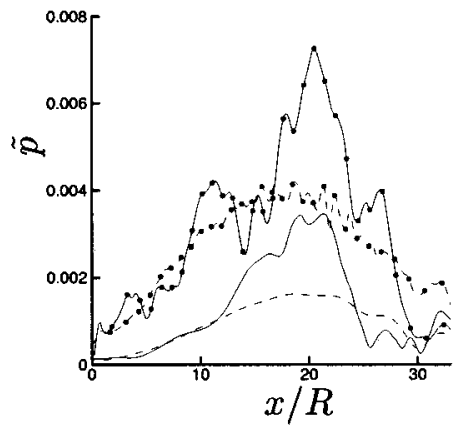

(b)

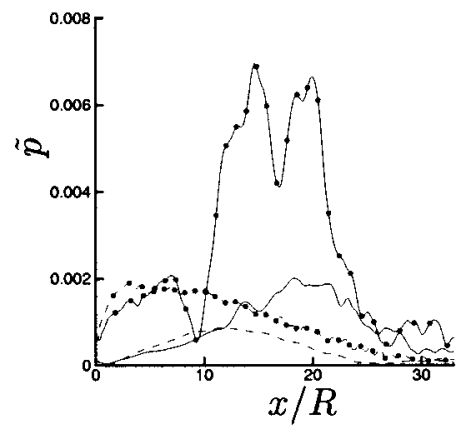

(e)

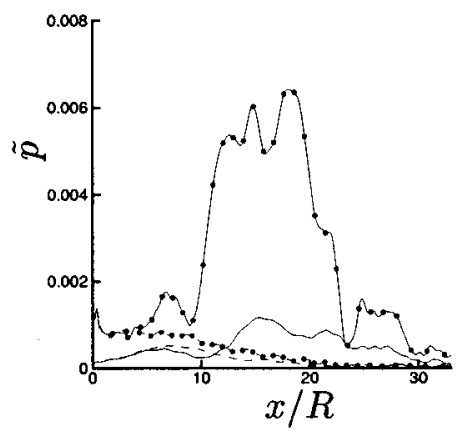

(h)

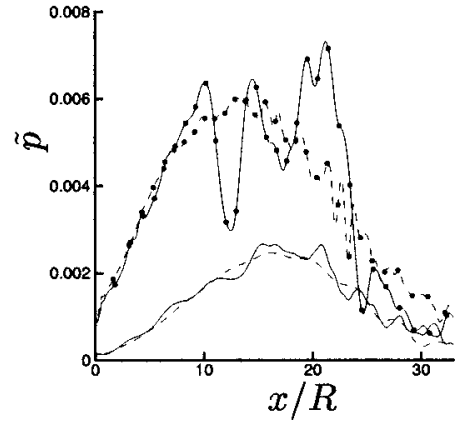

(c)

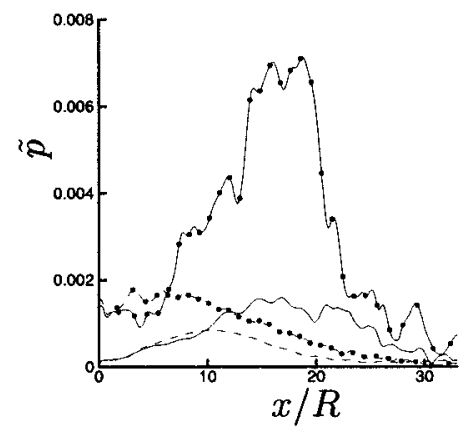

(f)

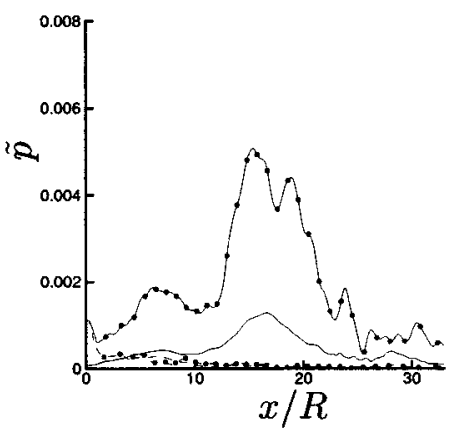

(i)

FIG. 6. Amplitude of the pressure Fourier coefficient for $n=1$ : (a) $\mathrm{St}=0.048$; (b) $\mathrm{St}=0.095$; (c) $\mathrm{St}=0.143$; (d) $\mathrm{St}=0.191$; (e) $\mathrm{St}=0.238$; (f) $\mathrm{St}=0.286$; (g) $\mathrm{St}=0.333$; (h) $\mathrm{St}=0.381$; (i) $\mathrm{St}=0.429$. The lines indicate: $--\mathrm{DNS}$ at $r=1 R$; ---- LNS at $r=1 R$; $-\mathrm{DNS}$ at $r=4 R$; ---- LNS at $r=4 R$.

LNS data were transformed to the frequency domain. The signal processing techniques used to transform the DNS are documented in the Appendix.

The spatial development of the pressure disturbances at various Strouhal numbers $\left(f D / U_{j}\right)$ are compared at two radial positions $(r=1 R$ and $r=4 R)$ for azimuthal modes $n$ $=0$ and $n=1$ in Figs. 5 and 6 , respectively. The pressure shown is $\widetilde{p}=|\hat{p}| / \rho a_{\infty}^{2}$, where $\hat{p}(x, y, \mathrm{St}, n)$ is the complex Fourier coefficient for the particular Strouhal number and azimuthal mode of interest. LNS and DNS agree (as indeed they must) near the inflow boundary (where they are forced with identical fluctuation field). Importantly, LNS provides an excellent representation of the growth and decay of mode $n=1$ at $\mathrm{St}=0.143$ [Fig. 6(c)], which is near the peak of the noise spectrum. Reasonable agreement between LNS and DNS is also seen for $n=1$ at Strouhal numbers near the peak in the range $0.0952 \leq \mathrm{St} \leq 0.1905$. This is similar to the experimental findings of Troutt and McLaughlin. ${ }^{5}$ However, for frequencies away from the peak in mode, the LNS fluctuations begin to decay closer to the inflow, and saturate with significantly lower amplitude than the corresponding fluctuations from DNS. Another trend in Fig. 6 is that the higher frequencies of the LNS calculation saturate earlier than the lower frequencies, an effect that is consistent with linear stability theory, but not evident in the DNS.

For all frequencies, but especially near the peak Strouhal number, the agreement between DNS and LNS is better for $n=1$ than it is for $n=0$. In DNS, both modes $n=0$ and $n$ $=1$ undergo similar amplification. In our simulations the azimuthal mode $n=0$, unlike mode $n=1$, does not have an extended region of amplification (see Figs. 5 and 6). The 
wave number spectrum of an instability wave at fixed frequency whose amplitude undergoes spatial growth and decay is broadband and not discrete, which might include supersonic phase velocities and consequently acoustic radiation. The more extended this region of amplification and decay the most likely to have a traveling wave with a significant supersonic phase velocity. To this end the azimuthal mode $n=1$ of the present jet is more likely to have significant linear noise sources, as is evident in Fig. 6. The azimuthal mode $n=0$, on the other hand, does not show an extended amplification region at the present jet Reynolds number and shear layer thickness. Consequently, the sound field in this case is dominated by the noise sources that are not present in the linear theory and LNS (see Fig. 5).

The observation that turbulence near the end of the potential core is responsible for a portion of the Mach wave radiation is not new. It was observed experimentally by Troutt and McLaughlin, ${ }^{5}$ whose near acoustic field measurements suggested that there were two distinct sources at a given frequency, one originating from the shear layer region, the other further downstream. In the present low Reynolds number simulation, the shear layers are initially thick enough that modes with frequencies above $\mathrm{St}=0.4$ are damped immediately, which we believe somewhat obscures this twosource effect. The noise from the shear layers and from further downstream cannot be clearly distinguished.

\section{DISCUSSION AND SUMMARY}

In this study we have evaluated the linear theory of Mach wave radiation in a perfectly expanded supersonic jet. The relative noise directivity predicted by linear computation was similar to that in the DNS, but the noise radiated by the first two modes in the linearized computation was substantially weaker. For the jet considered in this study, the first azimuthal mode agreed better with the DNS than the axisymmetric mode which was substantially underpredicted. At and near the peak Strouhal frequency, particularly for $n=1$, the amplification of disturbances in the LNS closely matched that in the DNS. However, for other frequencies the DNS data showed amplification rates comparable to those of the peak Strouhal number, whereas in the LNS data the disturbances away from the peak Strouhal number were damped. Except near the peak frequency, frequency modes in the DNS peaked in a region around and beyond the end of the potential core, which is further downstream than the corresponding peak in the LNS simulation.

Until present, evidence supporting the linear theory of Mach wave radiation was indirect: the general agreement of the directivity of the Mach wave radiation and its peak Strouhal number. While the theory is successful in predicting these, the present comparison shows that the noise generation process is not well modeled quantitatively by the linear theory, even when no further approximations (such as models of the incident turbulence or a slowly spreading mean flow) are made. For the jet considered in this study, nonlinear effects are not only present, but they dominate the noise process for the axisymmetric mode $n=0$, and contribute sig- nificantly to the $n=1$ mode at frequencies different from that of the peak radiation.

It is perhaps not surprising that the directivity of the acoustic field near the peak Strouhal number is insensitive to the details of the source process. Indeed, the growth and decay of any constant frequency convecting disturbance will produce such radiation, albeit at an amplitude that depends critically on the growth and decay rates of the disturbance with streamwise distance. The present results show that, except for the most amplified linear modes, the dominant sources arise further downstream and as the result of a nonlinear process, even though they produce similar overall directivity to the purely linear mechanism.

The need to include nonlinearity in order to correctly determine the spreading of the mean flow and set the growth and decay of the instability waves was recognized in the past. ${ }^{16}$ The results here suggest that, for all but the most amplified frequency and azimuthal mode, nonlinearity plays a more significant role in Mach wave radiation, because the mean flow spreading is inherently accounted for by using the mean flow predicted by the DNS in evolving the linearized disturbances. We are not able to ascertain, based on the present methodology, whether the differences between the linear and nonlinear computations it is due to a failure of LNS to correctly predict the amplification of disturbances in the near field (due to nonlinear saturation or interaction of the modes), or whether it is nonlinear mechanisms for sound radiation (or both). Such a distinction is important for future modeling efforts, and we hope to address it in future work.

\section{ACKNOWLEDGMENTS}

This research was supported by the National Science Foundation (T.C. and K.M.; CTS1501349) and NASA Glenn Research Center (J.B.F.).

\section{APPENDIX: SIGNAL PROCESSING TECHNIQUES}

This Appendix details methods for extracting frequency spectra from the data. The nature of data required that different methods be used for the DNS versus LNS.

\section{Discrete Fourier transform for LNS}

Since the LNS calculations are very nearly periodic, the Fourier spectra were calculated using standard discrete Fourier methods. Assuming a convection velocity of unity, the spatial periodicity of the inflow data was translated into a temporal periodicity with a period $T=21 R / a_{\infty}$. The total duration of the LNS data was to $3 T$, and it required only a single flow through time to reach an essentially time periodic condition. Our numerical experiments show that even one period of the LNS data is enough to accurately calculate the spectra at the smallest frequency, $f=a_{\infty} / 21 R$. The LNS data are sampled every time step to avoid aliasing. The resulting sampling rate was $f=100 a_{\infty} / R$, which is well above the maximum frequency considered in this study. 


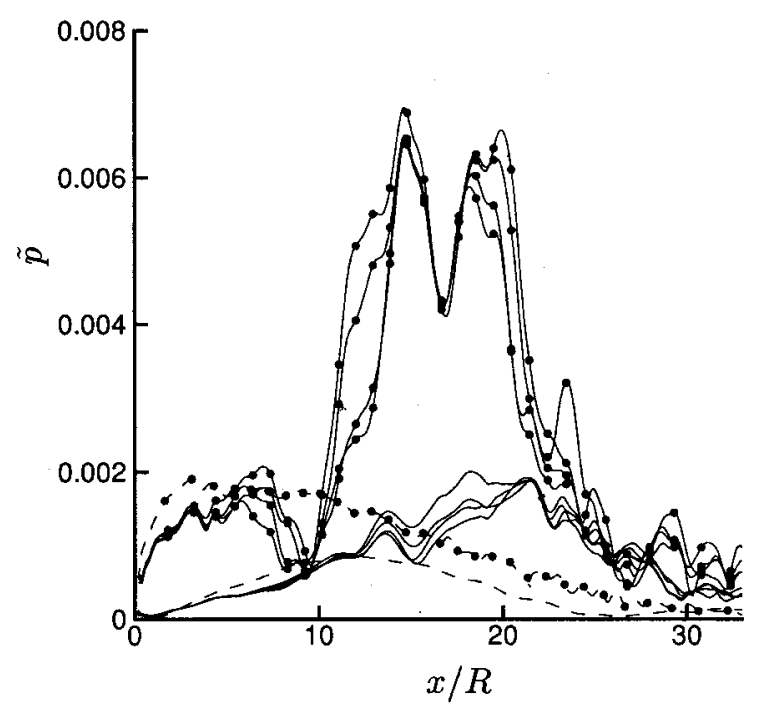

(a) $S t=0.238$

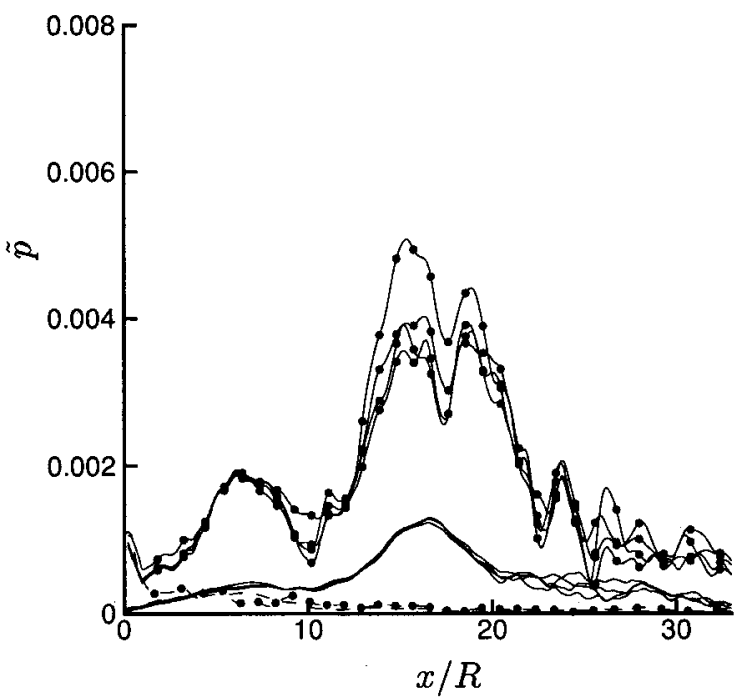

(b) $S t=0.428$

FIG. 7. Sensitivity of DNS pressure frequency spectra for $n=1$ to the period of the available data at $r=4 R$ and $r=1 R$. DNS spectra for periods $\mathrm{Ta}_{\infty} / R=9.4,9,8.5$, and $8 \times 21$. - - DNS at $r=1 R$; --@- LNS at $r$ $=1 R$; DNS at $r=4 R$; --- LNS at $r=4 R$.

\section{Lomb-Scargle periodogram for DNS}

A direct discrete Fourier transform would not be reliable in the case of DNS data because of several computational issues. Though the DNS data are quasi-periodic near the inflow, it is fully aperiodic further downstream. Thus imposing a periodicity here would contaminate the high frequencies. In addition, the DNS data were computed with a variable time step, and there were a few short-duration patches of missing DNS data. There are 2496 samples between computational times $156 R / a_{\infty}$ and $352 R / a_{\infty}$, constituting more than 9 periods of the inflow forcing.

A technique that is suited to unequally sampled data which may include missing values is the Lomb-Scargle periodogram. ${ }^{17,18}$ While a Fourier transform decomposes the time-series into a fundamental periodicity and a number of harmonics, a periodogram shows the power of each of these periodicities. A fast algorithm for computing the LombScargle spectrum was used. ${ }^{19}$

Since the sampling theorem applies only to evenly sampled data, the Nyquist frequency is not defined for the unevenly sampled DNS data. Nevertheless, an average Nyquist frequency can be defined as

$$
\bar{f}_{N}=\frac{1}{2 \overline{\Delta t}},
$$

where $\overline{\Delta t}$ is the average sampling interval. The average $\mathrm{Ny}$ quist frequency for the available DNS data is almost $13 a_{\infty} / R$, and is well above the highest frequencies considered in this study.

The sensitivity of the DNS frequency spectra to the period of the available data is presented in Fig. 7. While there are some fluctuations in the results by changing the period of the data the trends was well captured. All of the results presented in this study are calculated from data spanned over a period of $\mathrm{Ta}_{\infty} / R=9.4 \times 21$.

${ }^{1}$ D. G. Crighton and M. Gaster, "Stability of slowly diverging jet flow," J. Fluid Mech. 88, 397 (1976).

${ }^{2}$ M. Gaster, E. Kit, and I. Wygnanski, "Large-scale structures in a forced turbulent mixing layer," J. Fluid Mech. 150, 23 (1985).

${ }^{3}$ D. Oster and I. Wygnanski, "The forced mixing layer between parallel streams," J. Fluid Mech. 123, 91 (1982).

${ }^{4}$ C. K. W. Tam and D. Burton, "Sound generation by instability waves of supersonic flows. Part 2. Axisymmetric jets," J. Fluid Mech. 138, 273 (1984).

${ }^{5}$ T. R. Troutt and D. K. McLaughlin, "Experiments on the flow and acoustic properties of a moderate-Reynolds-number supersonic jet," J. Fluid Mech. 116, 123 (1982).

${ }^{6} \mathrm{C}$. K. W. Tam and P. J. Morris, "The radiation of sound by the instability waves of a compressible plane turbulent shear layer," J. Fluid Mech. 98, $349(1980)$.

${ }^{7}$ C. K. W. Tam, P. Chen, and J. M. Seiner, "Relationship between instability waves and noise of high-speed jets," AIAA J. 30, 1747 (1992).

${ }^{8}$ C. K. W. Tam and P. Chen, "Turbulent mixing noise from supersonic jets," AIAA J. 32, 1774 (1994).

${ }^{9}$ J. B. Freund, S. K. Lele, and P. Moin, "Direct simulation of a Mach 1.92 jet and its sound field," AIAA J. 38, 2023 (2000).

${ }^{10} \mathrm{~K}$. Mohseni, "A: Universality in vortex formation B: Evaluation of Mach wave radiation in a supersonic jet," Ph.D. thesis, California Institute of Technology, 2000.

${ }^{11} \mathrm{~S}$. K. Lele, "Compact finite difference schemes with spectral-like resolution," JETP 103, 16 (1992).

${ }^{12} \mathrm{~K}$. Mohseni and T. Colonius, "Numerical treatment of polar coordinate singularities," J. Comput. Phys. 157, 787 (2000).

${ }^{13}$ J. B. Freund, "A proposed inflow/outflow boundary condition for direct computation of aerodynamic sound," AIAA J. 35, 740 (1997).

${ }^{14}$ J. B. Freund, S. K. Lele, and P. Moin, "Compressibility effects in a turbulent annular mixing layer. Part 1. Turbulence and growth rate," J. Fluid Mech. 421, 229 (2000).

${ }^{15}$ D. Papamoschou and A. Roshko, "The compressible turbulent shear layer: An experimental study," J. Fluid Mech. 197, 453 (1988).

${ }^{16} \mathrm{P}$. J. Morris, "Flow characteristics of the large scale wavelike structures of a supersonic round jet," J. Sound Vib. 53, 223 (1977).

${ }^{17}$ N. R. Lomb, "Least-squares frequency analysis of unequally spaced data," Astrophys. Space Sci. 39, 447 (1976).

${ }^{18}$ J. D. Scargle, "Studies in astronomical time series analysis. II. Statistical aspects of spectral analysis of unevenly spaced data," Astrophys. J. 302, 757 (1982).

${ }^{19}$ W. H. Press, S. A. Teukolsky, W. T. Vetterling, and B. P. Flannery, Numerical Recipes, 2nd ed. (Cambridge University Press, Cambridge, 1992). 